Article

\title{
The Incompatibility of Pareto Optimality and Dominant-Strategy Incentive Compatibility in Sufficiently-Anonymous Budget-Constrained Quasilinear Settings
}

Rica Gonen ${ }^{1, *}$ and Anat Lerner ${ }^{2}$

${ }^{1}$ Department of Management and Economics, The Open University of Israel, 1 University Road, Raanana 43537, Israel

${ }^{2}$ Department of Mathematics and Computer Science, The Open University of Israel, 1 University Road, Raanana 43537, Israel; E-Mail: anat@ cs.openu.ac.il

* Author to whom correspondence should be addressed; E-Mail: gonenr@openu.ac.il; Tel.: +972 54 7278-563; Fax: +972-9-7780-668.

Received: 19 June 2013; in revised form: 2 November 2013 / Accepted: 7 November 2013 / Published: 18 November 2013

\begin{abstract}
We analyze the space of deterministic, dominant-strategy incentive compatible, individually rational and Pareto optimal combinatorial auctions. We examine a model with multidimensional types, nonidentical items, private values and quasilinear preferences for the players with one relaxation; the players are subject to publicly-known budget constraints. We show that the space includes dictatorial mechanisms and that if dictatorial mechanisms are ruled out by a natural anonymity property, then an impossibility of design is revealed. The same impossibility naturally extends to other abstract mechanisms with an arbitrary outcome set if one maintains the original assumptions of players with quasilinear utilities, public budgets and nonnegative prices.
\end{abstract}

Keywords: budget constraints; Pareto efficiency; incentive compatibility

\section{Introduction}

We study the limitations of the possibility space of deterministic, dominant-strategy incentive compatible, individually rational, and Pareto-optimal combinatorial auctions in a model with two 
players and two nonidentical items (four outcomes). Our model has multidimensional types, ${ }^{1}$ private values, nonnegative prices, and quasilinear preferences for the players with one relaxation: the players are subject to publicly known budget constraints. This setting is somewhat more complex than that of common auction literature as it adds budgets and heterogeneity, which more accurately describe mechanisms used in practice.

The investigated space better characterizes many real world problems such as commonly studied bandwidth (combinatorial) auctions. Consider the German and British $3 \mathrm{G}$ radio spectrum auctions in early 2000 where telecom companies bid so high as to have jeopardized their financial viability and consequently considerably slowed down capital investment in $3 \mathrm{G}$ equipment. Another contemporary example arises from globalized supply chains. Globalization has substantially increased competition among suppliers. As such, there are many suppliers who are trying to win business while incapable of delivering the contracted quantity/quality of procured goods.

The phenomena in the German and British $3 \mathrm{G}$ radio spectrum auctions as well as the present day proliferation of suppliers highlights the potential gap between willingness to pay and ability to pay, and the potential of better understanding how budget constraints affect auction design. Further consider that most goods are not sold in uniform bundles or used as single goods. Though blocks of radio bandwidth are apparently uniform they are not identical, as well can be said for goods in supply chain auctions which are bundled to fulfill diverse bill of materials. The addition of the seemingly minor dimension of heterogeneity profoundly affects auction design complexity.

Our result shows that the basic design requirements of dominant strategy incentive compatibility (DSIC), individual rationality (IR), and Pareto optimality (PO) lead to the presence of dictatorial mechanisms and these mechanisms can exhibit zero prices. This means a bandwidth auction can arise where one telecommunications company dictates its bandwidth allocation regardless of its competitors' values for the bandwidth and the government collects zero revenue. As the auctioneer would certainly take issue with such an outcome, in running such mechanism auctions one must consider an anonymity property that does not allow for a single designated competitor to dictate the auction and in turn the revenue.

A natural and weak requirement that prevents such dictatorial outcome is that in cases where the whole bandwidth is allocated to a single telecommunications company, then the company must value or be able to afford the bandwidth more than any other telecommunications company. With such a requirement no single telecommunications company can dictate the auction's allocation.

This motivates our discussion of how to go about designing a dominant-strategy incentive compatible combinatorial auction for allocating multiple nonidentical items in a Pareto-optimal manner when participants may be budget constrained and dictatorial design is ruled out. Our main result is an impossibility: in a model with multiple nonidentical items and nonarbitrary allocation of the all-item-bundle (a property we term nonarbitrary hoarding), there is no deterministic auction that is dominant-strategy incentive compatible, individually rational, and Pareto optimal. The same impossibility naturally extends to more abstract mechanisms with an arbitrary outcome set if

1 Multidimensional types, meaning that a player may have a separate arbitrary value for each of the four possible outcomes. 
one maintains the original assumptions of players with quasilinear utilities, public budgets, and nonnegative prices.

Our impossibility is composed of three parts. The first part proves under which conditions any mechanism $F$ that satisfies DSIC, IR, PO, and nonarbitrary hoarding will allocate singletons (an item for each player) and be a Vickrey-Clarke-Groves (VCG) [1-3] mechanism. The second part proves under which conditions any mechanism $F$ that satisfies the above properties will allocate singletons and be a "trivial pricing mechanism". A trivial pricing mechanism is a mechanism where one player is allocated a nonempty bundle for free regardless of the other players' valuations. The third part concludes our impossibility since both payments cannot coexist and maintain the dominant strategy incentive compatibility property.

Though our impossibility result does not require efficiency as a property, it does conclude the existence of efficient solutions. Nevertheless, since Pareto optimality and the property of nonarbitrary hoarding do not imply efficiency. Proving that VCG defines some of the mechanism space requires additional tools.

The tool we use to prove that VCG defines some of the mechanism space is a property we term stability. The stability property derives from the three properties of dominant-strategy incentive compatible, individually rational, and Pareto optimality together with nonarbitrary hoarding. Stability essentially bounds the prices of any such mechanism (with strictly positive prices) from below ensuring that no player has an incentive to collude with the auctioneer ex-post and offer the auctioneer side payments such that the colluding player can win all the items. To illustrate that stability can be used to bound the prices of any such mechanism from below consider one-item Vickrey auctions. In one-item Vickrey auctions the losing player cannot afford to make side payments to the auctioneer after the auction is concluded, in order to induce the auctioneer to give him the item instead of giving it to the winning player. In mechanisms that are not budget-constrained, ensuring that a player has no incentive to collude with the auctioneer ex-post to win all the items means that the price of the remaining items is at least his marginal value for the remaining items. In budget-constrained mechanisms it is sufficient to require that either the price of the remaining items is at least his remaining budget (after paying his allocation) or at least his marginal value for those items.

\subsection{Our Contribution}

Our result presents the following three contributions. First, that the general space of dominant strategy incentive compatible, individually rational, and Pareto-optimal combinatorial auctions with budgets includes dictatorial mechanisms. Second and most importantly, when dictatorial mechanisms are ruled out and a minimal property of anonymity (named nonarbitrary hoarding) is integrated, the design of the above combinatorial auctions becomes an impossibility. Moreover our analysis concludes the existence of efficient and inefficient solution domains which consequently leads to an impossibility as a manipulation between an efficient and inefficient domain can occur. Third, the above three properties together with a natural anonymity assumption, i.e. nonarbitrary allocation of the all-item-bundle, imply the useful property of player-auctioneer coalition resilience even when side payments are allowed. We 
termed the player-auctioneer coalition resilience property stability and use it to prove VCG pricing when paid prices are all positive.

\subsection{The Role of Determinism and Asymmetry from Heterogeneity}

Our result shows the impossibility of designing budget-constrained combinatorial auctions that are dominant-strategy incentive compatible, individually rational, Pareto optimal and nonarbitrary hoarding in a model of two heterogeneous items. As the nature of the result is negative it applies to any more complex models that include heterogeneous items. Nevertheless it is easy to see that if one substitutes the two heterogeneous items with two homogeneous items all the claims still hold in a similar manner. That is to say that the introduction of asymmetry by the heterogeneity of the items does not change the nature of the results, claims, or proofs. Therefore for simplicity the result could be presented in the model of two homogeneous items. However there is much benefit in presenting the impossibility result in the heterogeneous model. Though the nature of the result is negative the structure of the proofs gives insights into which domains of valuations will allow the existence of an efficient solution and which domains will necessitate an inefficient solution. If we chose to present the impossibility in the homogeneous model the conclusion we would derive regarding the domains of efficiency and inefficiency would not necessarily be applicable to the heterogeneous model and would provide a much narrower view of the problem at hand.

Throughout the paper we assume deterministic mechanisms. To understand the role of determinism in our result one must look into the literature of nondeterministic constrained auctions such as [4]. [4]'s work defines the properties of constrained-efficient auctions, i.e., maximizing the expected social welfare under Bayesian incentive compatibility and budget-constrained players. [4] states that the domains of efficiency and inefficiency are determined by a threshold value. The computation of the threshold value makes use of expectation and allows for allocations with negative utility for the players. Therefore [4]'s threshold cannot be used in an individually rational deterministic setting. The domains of efficiency and inefficiency that can be concluded from our analysis are determined by the smaller of the budgets. Meaning that the smaller budget defines the threshold for inefficiency. The immediate implication of the smaller budget as the threshold of inefficiency is that the smaller budget cannot be a privately known value but must be publicly known. As such, in our deterministic setting both budgets are publicly knownmuch like [5,7]; while in [4] and [8]'s nondeterministic setting the budgets are privately known. ${ }^{2}$ Another implication of determinism on our result is that individual rationality is required for each allocation. Therefore an auction that charges losing players such as an all-play-auction cannot fulfill the individual rationality property. Consequently there is a gap in price between winning and losing and thus a manipulation between an efficient and inefficient domain can occur, leading to an impossibility.

2 Since the budgets can be equal, nonarbitrary hoarding together with dominant-strategy incentive compatibility imply that the second budget has to be public knowledge if the first budget is. 


\subsection{Prior Literature}

It is well known that in quasilinear environments with a complete preference domain over at least three outcomes and non-constrained players, only VCG mechanisms satisfy the dominant-strategy incentive compatible property [9]. ${ }^{3}$ Nevertheless when preferences are subject to free disposal and no externalities are assumed, as is common in combinatorial auctions, then the possibility space of dominant-strategy incentive-compatible combinatorial auctions in the multidimensional type model is not defined. Several papers investigate computationally feasible dominant-strategy incentive-compatible (but inefficient) auctions with one-dimensional private-values $[12,13]$ as well as with multidimensional private-value settings with some additional restrictions on the preference space $[14,15]$.

In recent years, several papers studied budget-constrained combinatorial auctions. The authors of [16] characterized some of the space of deterministic, dominant-strategy incentive compatible, individually rational and Pareto optimal budget-constrained combinatorial auctions and showed that the space studied essentially includes one type of mechanism that behaves dictatorially. The authors of [6] showed that there does not exist a deterministic auction that is individually rational, dominant-strategy incentive compatible and Pareto optimal with potentially negative prices and privately known budgets, even when players are one-dimensional types. The authors of [7] showed that the same impossibility holds for one-dimensional types with different items and publicly known multi-item demand. The authors of [5] also showed the same impossibility with publicly known budgets if multidimensional types (two identical items with three outcomes) are considered.

[5-7] allow negative prices to exist, i.e., some players are paid for participation in the auction either by the mechanism or by the other players. Practical auction implementations such as the FCC bandwidth auction usually cannot afford or are unwilling to consider paying bidders (telecommunications companies) for their participation nor are they interested in encouraging side payments among the participants. Therefore similar to [4]'s model we chose to assume that all prices are nonnegative. The assumption that all prices are nonnegative narrows down the domain of possible allocations in comparison with the potential negative prices model with multidimensional types. Nevertheless some of the the mechanisms which fulfill the three properties of dominant strategy incentive compatible, individually rational, and Pareto optimal in the nonnegative price model are not included in the mechanism space that fulfills the same properties in the negative price model. The reason for the above is the property of Pareto optimality. Since the nonnegative prices model has a smaller set of possible allocations there exist situations where a mechanism does not fulfill the Pareto optimal property in the model with negative prices but does fulfill the Pareto optimal property in the nonnegative price model.

[6] also characterizes the possibility space of dominant-strategy incentive compatibility and Pareto optimal budget-constrained combinatorial auction mechanisms, [6]'s characterization is restricted to one-dimensional types and therefore their possibility space characterization does not imply the possibility space in our model with multidimensional types. More specifically, [6] showed that for

3 In quasilinear environments only Groves' mechanisms satisfy the dominant-strategy incentive compatible and Pareto optimal properties $[10,11]$. 
multi-unit demand and identical items, Ausubel's clinching auction, which assumes public budgets and additive valuations, uniquely satisfies the properties (described above). In a similar model with small randomized modification [8] showed that [6]'s result can be obtained with private budgets. Similarly Ausubel's clinching auction was concluded by [7] for one-dimensional types with different items and publicly known multi-item demand. For unit-demand players with private values and budget constraints in the one-dimensional types model there are several deterministic mechanisms that fulfill the properties of incentive compatible and Pareto optimality (see [17,18]). In nondeterministic mechanisms with a one-dimensional types model (one indivisible unit) [4] characterizes constrained-efficiency mechanisms, which are mechanisms that maximize the expected social welfare under Bayesian incentive compatibility and budget constraints in a nonnegative price model. [4]'s results are not an impossibility as per the nature of nondeterministic mechanisms, though the characterization points out the limitations in designing mechanisms with budget-constrained players, even in the probabilistic domain. ${ }^{4}$

There are few other works that focus on revenue maximization under budget constraints. The authors of $[19,20]$ analyze how budgets change the classic results on "standard" auction formats, showing, for example, that first-price auctions raise more revenue than second-price auctions when bidders are budget-constrained and that the revenue of a sequential auction is higher than the revenue of a simultaneous ascending auction. The authors of [21,22] construct single-item auctions that maximize the seller's revenue.

\subsection{Paper Organization}

The paper is organized as follows. Notation and definitions are presented in Section 2. Section 3 discusses the presence of dictatorial mechanisms when designing deterministic, dominant-strategy incentive compatible, individually rational, Pareto optimal budget-constrained combinatorial auctions and defines the anonymity property of nonarbitrary hoarding. Section 4 shows the implications of the properties discussed above and proves VCG pricing for any nontrivial pricing mechanism that satisfies the four properties above. Section 5 proves our main result, the impossibility of design. The appendix presents some of the technical proofs.

\section{Notation and Definitions}

We consider combinatorial auction mechanisms with two heterogeneous items and two players. Let $N=\{1,2\}$ be the set of players and $C=\left\{c_{1}, c_{2}\right\}$ be the set of items. Let $\mathcal{B}$ be the set of all subsets of items, that is, $\mathcal{B}=2^{C}=\left\{\emptyset,\left\{c_{1}\right\},\left\{c_{2}\right\},\left\{c_{1}, c_{2}\right\}\right\}$.

Each player, $i$, has a private value, $v_{i}(B)$, for every bundle $B \in \mathcal{B}$ drawn from a valid valuation space $\mathcal{V}_{i}$, i.e., players are multi-minded and have different private values for different bundles of the items. ${ }^{5}$ We denote player $i$ 's private values by a triple: $V_{i}=\left(v_{i}\left(c_{1}\right), v_{i}\left(c_{2}\right), v_{i}\left(c_{1}, c_{2}\right)\right) \in \mathcal{V}_{i}$. We assume that $v_{i}(\emptyset)=0$, that is, for both players, the valuation

4 See discussion on the role of determinism in our work.

5 Throughout the paper, we consider valuation spaces where not all valuations are included in the valuation space. 
of the empty bundle is zero. We also assume that $v_{i}\left(B^{\prime}\right) \leq v_{i}(B)$ whenever $B^{\prime} \subseteq B$, i.e., free disposal; for both players, the allocation of an extra item cannot reduce their valuation (the usual assumption in combinatorial auctions). As players are multi-minded and have different private values for different bundles of the items, a player, $i$, may have a separate arbitrary value for each of the four possible outcomes, meaning our valuation space is a multidimensional valuation space and the players have multidimensional-type valuations.

We assume that the players have limited budgets, $b_{1}$ and $b_{2}$ respectively, for acquiring items and these budgets are publicly known information. Without loss of generality we assume that $b_{1} \leq b_{2}$.

We denote the auction mechanism $F\left(V_{1}, V_{2}, b_{1}, b_{2}\right)=\left(B_{1}, B_{2}, p\left(B_{1}\right), p\left(B_{2}\right)\right)$ where $B_{i}$ is the bundle allocated to player $i$; and $p\left(B_{i}\right)$ is the price of bundle $B_{i}$. We assume that all the prices are nonnegative, i.e., $p\left(B_{i}\right) \geq 0$ for $i \in\{1,2\}$.

DEFINITION 1 Player 1, player 2, and the auctioneer's utility are defined as follows: Player i's utility is

$$
u_{i}\left(F\left(V_{1}, V_{2}, b_{1}, b_{2}\right)\right)= \begin{cases}v_{i}\left(B_{i}\right)-p\left(B_{i}\right) & \text { if } p\left(B_{i}\right) \leq b_{i} \\ -\infty & \text { otherwise }\end{cases}
$$

The auctioneer's utility is $u_{a}\left(F\left(V_{1}, V_{2}, b_{1}, b_{2}\right)\right)=p\left(B_{1}\right)+p\left(B_{2}\right)$.

For simplicity of notation, whenever $F, V_{1}, V_{2}, b_{1}$, and $b_{2}$ are clear from the context we will denote $u_{i}\left(F\left(V_{1}, V_{2}, b_{1}, b_{2}\right)\right)$ by $u_{i}$.

\section{DEFINITION 2 Determinism}

An auction mechanism $F\left(V_{1}, V_{2}, b_{1}, b_{2}\right)$ is called deterministic iffor every given input it outputs a single outcome.

\section{Definition 3 Trivial Pricing Mechanism}

We say that a mechanism $F$ is a trivial pricing mechanism if there is an input $\left(V_{1}, V_{2}, b_{1}, b_{2}\right)$, such that $F\left(V_{1}, V_{2}, b_{1}, b_{2}\right)=\left(B_{1}, B_{2}, p\left(B_{1}\right), p\left(B_{2}\right)\right)$ and $\exists i \in\{1,2\}$ s.t. $\left(B_{i} \neq \emptyset\right.$ and $\left.p\left(B_{i}\right)=0\right)$. That is, there is a player $i$ who is allocated a nonempty bundle for free regardless of the other player's valuations.

\section{DEFINITION 4 Efficiency}

Let $F\left(V_{1}, V_{2}, b_{1}, b_{2}\right)=\left(B_{1}, B_{2}, p\left(B_{1}\right), p\left(B_{2}\right)\right)$ be an auction mechanism. Denote by $L=\left\{\left(B^{\prime}, B^{\prime \prime}\right) \mid B^{\prime} \in\right.$ $\mathcal{B}$ and $B^{\prime \prime} \in \mathcal{B}$ and $\left.B^{\prime} \cap B^{\prime \prime}=\emptyset\right\}$. We say that $F$ is efficient if for any $V_{1}, V_{2}$ :

$\left(B_{1}, B_{2}\right) \in \operatorname{argmax}_{\left(B^{\prime}, B^{\prime \prime}\right) \in L}\left(v_{1}\left(B^{\prime}\right)+v_{2}\left(B^{\prime \prime}\right)\right)$.

\section{DEFINITION 5 Dictatorship}

An auction mechanism $F\left(V_{1}, V_{2}, b_{1}, b_{2}\right)$ is called dictatorial if there exists a player $i \in\{1,2\}$ (the dictator) such that for every $V_{i}, V_{\hat{i}}, V_{\hat{i}}^{\prime}, u_{i}\left(F\left(V_{i}, V_{\hat{i}}, b_{1}, b_{2}\right)\right)=u_{i}\left(F\left(V_{i}, V_{\hat{i}}^{\prime}, b_{1}, b_{2}\right)\right)$.

Intuitively, a mechanism is called dictatorial if there is a player $i$ such that the valuations of the other player $\hat{i}$ cannot affect his utility. Note that if the dictator $i$ is indifferent to whether he receives one allocation or another then player $\hat{i}$ 's valuations can affect the output.

We next define three properties: individual rationality (IR), Pareto optimality (PO) and dominant strategy incentive compatibility (DSIC). 


\section{Definition 6 Property 1: Individual Rationality (IR)}

An auction mechanism $F\left(V_{1}, V_{2}, b_{1}, b_{2}\right)$ is called individually rational if for every player $i$, $u_{i}\left(F\left(V_{1}, V_{2}, b_{1}, b_{2}\right)\right) \geq 0$. That is, for each player $i$ :

$p\left(B_{i}\right) \leq \min \left\{b_{i}, v_{i}\left(B_{i}\right)\right\}$ (IR of player $i$ )

Note that the auctioneer's utility is nonnegative from our assumption that all the prices are nonnegative.

\section{Definition 7 Property 2: Pareto Optimality (PO)}

An auction mechanism $F$ is called Pareto optimal if for every input $V_{1}, V_{2}, b_{1}, b_{2}$, such that $V_{1}, V_{2}$ in $\mathcal{V}_{1} \times \mathcal{V}_{2}$, there is no auction mechanism $F^{\prime}$ such that all the following inequalities hold, with at least one strong inequality:

- $u_{1}\left(F^{\prime}\left(V_{1}, V_{2}, b_{1}, b_{2}\right)\right) \geq u_{1}\left(F\left(V_{1}, V_{2}, b_{1}, b_{2}\right)\right)$

- $u_{2}\left(F^{\prime}\left(V_{1}, V_{2}, b_{1}, b_{2}\right)\right) \geq u_{2}\left(F\left(V_{1}, V_{2}, b_{1}, b_{2}\right)\right)$

- $u_{a}\left(F^{\prime}\left(V_{1}, V_{2}, b_{1}, b_{2}\right)\right) \geq u_{a}\left(F\left(V_{1}, V_{2}, b_{1}, b_{2}\right)\right)$

\section{DEFINITION 8 Property 3: Dominant Strategy Incentive Compatibility (DSIC)}

An auction mechanism $F\left(V_{1}, V_{2}, b_{1}, b_{2}\right)$ is called dominant strategy incentive compatible (DSIC) if neither of the two players can increase his own utility by reporting false valuations. That is, given the true valuations $V_{1}$ and $V_{2}$ in $\mathcal{V}_{1} \times \mathcal{V}_{2}$, for every $V_{1}^{\prime}$ and $V_{2}^{\prime}$ in $\mathcal{V}_{1} \times \mathcal{V}_{2}$ the following hold:

- $u_{1}\left(F\left(V_{1}, V_{2}^{\prime}, b_{1}, b_{2}\right)\right) \geq u_{1}\left(F\left(V_{1}^{\prime}, V_{2}^{\prime}, b_{1}, b_{2}\right)\right)$

- $u_{2}\left(F\left(V_{1}^{\prime}, V_{2}, b_{1}, b_{2}\right)\right) \geq u_{2}\left(F\left(V_{1}^{\prime}, V_{2}^{\prime}, b_{1}, b_{2}\right)\right)$

Note that if a given mechanism that satisfies PO does not allocate all the items, then we can conclude that the players are indifferent to the free addition of items that were left out of the allocation. From free disposal we know that the addition of items cannot decrease any player's utility. Therefore, for any mechanism $F$ that satisfies the properties and does not allocate all the items, there is a mechanism $F^{\prime}$ that satisfies the properties and allocates all the items, such that $F^{\prime}$ is identical to $F$ except for the allocation of the items not allocated in $F$. Moreover $F^{\prime}$ can be generated from $F$ by the following procedure. If $\bigcup_{i \in N} B_{i} \neq C$ then randomly allocate the items in $C-\bigcup_{i \in N} B_{i}$ to the two players with no extra charge. Thus we consider only mechanisms in which all items are allocated, $\bigcup_{i_{\in} N} B_{i}=C$.

\section{The Presence of Dictatorial Mechanisms}

In this section we prove that the requirement for DSIC, IR, and PO leads to the presence of dictatorial mechanisms with zero prices. We show that there exists a deterministic dictatorial combinatorial auction mechanism with (publicly known) budget-constrained players that satisfies the properties of DSIC, IR, and PO.

PROPOSITION 1 The following dictatorial mechanism with zero prices, an indifference breaking rule, and player $i$ as the dictator satisfies the properties of IR, DSIC, and PO.

Denote by Best ${ }_{i}=\left\{B \in \mathcal{B} \mid v_{i}\left(c_{1}, c_{2}\right)=v_{i}(B)\right\}$. $p\left(B_{1}\right)=p\left(B_{2}\right)=0$. 
If $\mid$ Best $_{i} \mid=1$ then $B_{i}=\left\{c_{1}, c_{2}\right\}$

else choose a bundle $B_{i}$ from Best $t_{i}$ such that $v_{\hat{i}}\left(C-B_{i}\right)$ is maximized.

Proposition 1's proof can be found in Appendix A.

In the above proposition, we proved that there exists a dictatorial mechanism that satisfies DSIC, IR and PO. In the next proposition, we further prove that any dictatorial mechanism that fulfills these properties must have zero prices.

PROPOSITION 2 Let $F$ be a dictatorial mechanism that satisfies the three properties of IR, DSIC and $P O$. Let $i$ be the dictator and let $\hat{i}$ be the other player. Then it must be the case that $p\left(B_{i}\right)=p\left(B_{\hat{i}}\right)=0$.

Proposition 2's proof can be found in Appendix B.

The above proposition leads to the question of how to rule out the dictatorial mechanisms from the domain of possible mechanisms. In other words, can one require a minimal property that will rule out dictatorships? Fortunately the answer to the above question is positive. We provide the following natural and weak requirement. In cases where the all-item-bundle is allocated to a single player the winner must value or afford the all-item-bundle more than the losing player. With this requirement no single player can dictate the auction allocation. We term the above described anonymity property nonarbitrary hoarding. In the rest of the paper we show that if we rule out dictatorships by adding the weak property of nonarbitrary hoarding an impossibility of design is revealed. The formal definition of nonarbitrary hoarding follows.

\section{DEFINITION 9 Property 4: Nonarbitrary Hoarding}

Denote the two players by player $i$ and player $\hat{i}, i \neq \hat{i}$. We say that an auction mechanism $F$ obeys nonarbitrary hoarding if the following condition holds for every player $i \in\{1,2\}$ :

If $B_{i}=\left\{c_{1}, c_{2}\right\}$ then $\min \left\{b_{\hat{i}}, v_{\hat{i}}\left(c_{1}, c_{2}\right)\right\} \leq \min \left\{b_{i}, v_{i}\left(c_{1}, c_{2}\right)\right\}$.

Intuitively, a mechanism fulfills nonarbitrary hoarding if whenever both items are allocated to a single player the player is chosen nonarbitrarily, i.e. in accordance to the valuations and budgets of the two players. Furthermore the player chosen has to be able to afford the two-item bundle more than the other player.

To better understand the property of nonarbitrary hoarding consider the extended setting of a $k$-item auction mechanism. The nonarbitrary hoarding property states that the $k$-item bundle is not allocated arbitrarily, that is:

If $B_{i}=\left\{c_{1}, \cdots, c_{k}\right\}$ then $\min \left\{b_{i}, v_{i}\left(B_{i}\right)\right\} \geq \min \left\{b_{\hat{i}}, v_{\hat{i}}\left(B_{i}\right)\right\}$.

Note that the property of nonarbitrary hoarding does not imply that a nonarbitrary hoarding mechanism has to allocate the two-item bundle to one of the players under some valuations and budgets.

In Proposition 1 we provide a mechanism that satisfies the properties of IR, DSIC, and PO. This mechanism is not nonarbitrary hoarding, as there is one player, player 1, who is always allocated his best choice regardless of the other player's valuations.

In the rest of the paper we prove that it is impossible to design deterministic, dominant-strategy incentive compatible, individually rational, Pareto optimal and nonarbitrary hoarding combinatorial auctions with (publicly known) budget-constrained players. 


\section{The Impossibility Theorem}

\subsection{Implications of Properties}

In this subsection we define a new property called stability and show that stability is implied from the properties IR, nonarbitrary hoarding, PO, and DSIC in any nontrivial pricing mechanism. We then use the stability property to derive the set of prices for any nontrivial pricing mechanism that satisfies these four properties.

\subsubsection{Stability}

\section{DEFINITION 10 Stability}

Let $i$ and $\hat{i}, i \neq \hat{i}$ denote the two players. An auction mechanism is stable if for every player $i$ and for any $V_{i} \in \mathcal{V}_{i}, V_{\hat{i}} \in \mathcal{V}_{\hat{i}}$ the following condition holds:

$p\left(B_{\hat{i}}\right) \geq \min \left\{v_{i}\left(c_{1}, c_{2}\right)-v_{i}\left(B_{i}\right), b_{i}-p\left(B_{i}\right)\right\}$ (stability of player $i$ )

Stability means that no player $i$ can collude with the auctioneer (or defect with the auctioneer) against player $\hat{i}$ to receive the two-item bundle. That is, player $i$ cannot simultaneously increase the auctioneer's utility (by $\varepsilon$ side payment) while maintaining a higher utility from the two-item bundle than the utility from the mechanism's allocation. The above intuition can be derived for player $i$ by subtracting $p\left(B_{i}\right)$ from both sides of player $i$ 's stability inequality in the case where budget is not a limitation, i.e., $p\left(B_{\hat{i}}\right) \geq$ $v_{i}\left(c_{1}, c_{2}\right)-v_{i}\left(B_{i}\right) \Rightarrow v_{i}\left(B_{i}\right)-p\left(B_{i}\right) \geq v_{i}\left(c_{1}, c_{2}\right)-\left(p\left(B_{i}\right)+p\left(B_{\hat{i}}\right)\right)$. The intuition above also holds for player $i$ when budget is his limitation, i.e., $v_{i}\left(c_{1}, c_{2}\right)-v_{i}\left(B_{i}\right)>b_{i}-P\left(B_{i}\right) \Rightarrow p\left(B_{i}\right)+p\left(B_{\hat{i}}\right) \geq b_{i}$.

While in mechanisms that are not budget constrained, ensuring that a player has no incentive to collude with the auctioneer ex-post to win all the items means that the price of the remaining items is at least his marginal value for the remaining items; in budget-constrained mechanisms it is sufficient to require that either the price of the remaining items is at least his remaining budget (after paying his allocation) or his marginal value for those items.

In Appendix $\mathrm{C}$ we show that stability holds in any nontrivial pricing mechanism that satisfies the four properties of IR, PO, nonarbitrary hoarding and DSIC.

We prove Lemma C. 1 by considering the different feasible allocations. We show for every allocation, that if stability does not hold then one of the properties of: IR, PO, nonarbitrary hoarding, and DSIC is violated, or the mechanism must be a trivial pricing mechanism.

The proof of Lemma C.1 can be found in Appendix C.

\subsubsection{Determining the Prices}

In the following proposition we prove the price structure of any nontrivial pricing mechanism that satisfies the four properties. In Lemma C.1 we proved that any nontrivial pricing mechanism that satisfies the four properties must satisfy stability. In Definition 10 (Stability) we stated the prices' lower bound. ${ }^{6}$

6 In cases of singleton allocations $p\left(B_{2}\right)=b_{1}-p\left(B_{1}\right)$ and $p\left(B_{1}\right)=b_{2}-p\left(B_{2}\right)$ implies a trivial pricing mechanism. 
We now show that the prices cannot be higher than the lower bound and therefore they must equal the lower bound.

PROPOSITION 3 In any nontrivial pricing mechanism that satisfies the properties of IR, DSIC, PO, and nonarbitrary hoarding, the prices must be as follows:

1. If $B_{i}=\left\{c_{1}, c_{2}\right\}$ and $B_{\hat{i}}=\emptyset$ then:

$$
p\left(B_{i}\right)=\min \left\{b_{\hat{i}}, v_{\hat{i}}\left(c_{1}, c_{2}\right)\right\} \text { and } P\left(B_{\hat{i}}\right)=0 \text {. }
$$

2. Else (singletons allocation):

$$
p\left(B_{i}\right)=v_{\hat{i}}\left(c_{1}, c_{2}\right)-v_{\hat{i}}\left(B_{\hat{i}}\right) \text {. }
$$

Proposition 3's proof can be found in Appendix D.

In Appendix E we analyze the implications of the PO property on the allocation of the two-item bundle to one player. In the following section we use the implications of PO to show that there is no mechanism $F\left(V_{1}, V_{2}, b_{1}, b_{2}\right)$ that satisfies the four properties.

\subsection{Impossibility Statement}

In this subsection we show that for any given $V_{1}, V_{2}, b_{1}$ and $b_{2}$ there is no mechanism $F\left(V_{1}, V_{2}, b_{1}, b_{2}\right)$ that satisfies the four properties of IR, DSIC, PO, and nonarbitrary hoarding.

In Appendix F, Lemma F.1 we show under which conditions any mechanism $F$ that satisfies the properties will allocate singletons and be a trivial pricing mechanism. In Appendix F, Lemma F.2 we show under which conditions any mechanism $F$ that satisfies the properties will allocate singletons and be a VCG mechanism. Proposition 1 then concludes that the two lemmas (Lemmas F.1 and F.2) cannot coexist.

THEOREM 1 There is no mechanism $F\left(V_{1}, V_{2}, b_{1}, b_{2}\right)$ that satisfies IR, DSIC, PO, and nonarbitrary hoarding for any given $V_{1}, V_{2}, b_{1}, b_{2}$.

Theorem 1's proof can be found in Appendix G.

\section{Concluding Remarks}

We analyze the space of deterministic, dominant-strategy incentive compatible, individually rational, and Pareto optimal combinatorial auctions with (publicly known) budget-constrained players. Our model has multidimensional types, nonidentical items, private values, and quasilinear preferences for the players. We showed that the above space includes dictatorial mechanisms that exhibit zero prices. Interestingly we proved that if dictatorial mechanisms are ruled out by the natural anonymity property of nonarbitrary hoarding then an impossibility of design is revealed. The minimality of the nonarbitrary hoarding property in creating the impossibility is the topic of our research in progress.

Though the nature of our result is an impossibility, Lemma F.1 and Lemma F.2 provide insights into which valuation domains will allow the existence of efficient solutions and which domains will enforce inefficient solutions. Additionally, Lemma F.1 and Lemma F.2 establish the publicly-known budget $b_{1}$ as the threshold for efficiency. 
[5-7] allow negative prices to exist, i.e., some players are paid for participation in the auction either by the mechanism or by the other players. Auction holders such as the FCC would find it impractical and undesirable or are unwilling to pay bidders (telecommunications companies) for their participation, nor are they interested in encouraging side payments among the participants. Therefore, similar to [4]'s model we chose to assume that all prices are nonnegative in our multidimensional type model. On the one hand the assumption that all prices are nonnegative narrows the domain of possible allocations in comparison with the allowed negative prices model. On the other hand narrowing the domain of possible allocations allows for more mechanisms to fulfill DSIC, IR, and in particular the Pareto optimality property. In our work with nonidentical items and multidimensional types, narrowing the domain of possible allocations exposed the existence of dictatorial mechanisms and the design impossibility once an anonymity property was assumed. An interesting outcome of our result would be to revisit the other budget-constrained combinatorial auction literature such as [6,7] and narrow the domain of possible allocations to determine the role of the nonarbitrary hoarding property in the impossibility of design of the one-dimensional types model.

\section{Appendix}

\section{A. Proposition 1's Proof}

PROOF OF PROPOSITION 1 The mechanism satisfies IR as each player pays zero, that is, no player pays more than his budget or his values. The mechanism satisfies $P O$, as player $i$ is allocated his most valued bundle for free. As we assume nonnegative prices, there is no other allocation that can increase his utility. Player $\hat{i}$ is allocated the most valued bundle among player $i$ 's best choice; any increase in player $\hat{i}$ 's utility will strictly decrease the utility of player $i$. The mechanism is DSIC as player $i$ is allocated his best choice for free, so any deviation can only decrease his utility irrespective of player $\hat{i}$ 's reported valuation. Player $\hat{i}$ is allocated the highest valued bundle from a limited set of bundles that does not depend on his declarations. Any deviation can only decrease his utility.

\section{B. Proposition 2's Proof}

ProOF OF PROPOSITION 2 Let $F$ be a dictatorial mechanism that satisfies the three properties of IR, DSIC and PO. Let $i$ be the dictator and let $\hat{i}$ be the other player. Then:

1. $p\left(B_{\hat{i}}\right)=0$, regardless of the bundle allocated to the non-dictator $\hat{i}$

Assume to the contrary that $p\left(B_{\hat{i}}\right)=y>0$. From player $\hat{i}$ 's DSIC we know that $p\left(B_{\hat{i}}\right)$ cannot be a function of $\hat{i}$ 's valuations. From the definition of dictatorship 5 and the dictator's DSIC we conclude that the allocation of a DSIC dictatorial mechanism is chosen to maximize the dictator's utility. Thus it might be the case that the chosen allocation is not IR for player $\hat{i}$ as $v_{\hat{i}}\left(B_{\hat{i}}\right)<y$.

2. If $\left|B_{i}\right|=1$, i.e., the dictator is allocated a singleton, then $p\left(B_{i}\right)=0$.

Following the definition of dictatorship 5, the price paid by player $i$ cannot depend on the other player's valuations and following player i's DSIC, the price he pays cannot depend on his own 
valuations. Thus, we then conclude that player i's price must be a constant. Suppose that $p\left(B_{i}=\left\{c_{1}\right\}\right) \neq p\left(B_{i}^{\prime}=\left\{c_{2}\right\}\right)$ and, without the loss of generality, assume that $p\left(B_{i}=\left\{c_{1}\right\}\right)>$ $p\left(B_{i}^{\prime}=\left\{c_{2}\right\}\right)$. Consider the following situation:

$v_{i}\left(c_{1}, c_{2}\right)-p\left(B_{i}^{\prime \prime}=\left\{c_{1}, c_{2}\right\}\right)<v_{i}\left(c_{1}\right)-p\left(B_{i}=\left\{c_{1}\right\}\right)<v_{i}\left(c_{2}\right)-p\left(B_{i}^{\prime}=\left\{c_{2}\right\}\right), v_{i}\left(c_{1}\right)>v_{i}\left(c_{2}\right)$ and $v_{\hat{i}}\left(c_{2}\right)>v_{\hat{i}}\left(c_{1}\right)$. From the definition of dictatorship we conclude that the allocation must be $B_{i}^{\prime}=\left\{c_{2}\right\}$ as to maximize player $i$ 's utility. However, this allocation is not PO as player $i$ prefers the other singleton, i.e. $B_{i}=\left\{c_{1}\right\}$ for the same price as in allocation $B_{i}^{\prime}$ and player $\hat{i}$ prefers the allocation of item $c_{2}$ for the same price as in allocation $B_{\hat{i}}^{\prime}$.

Now suppose to the contrary that $p\left(B_{i}=\left\{c_{1}\right\}\right)=p\left(B_{i}^{\prime}=\left\{c_{2}\right\}\right)=y>0$. Consider the following valuations: $v_{i}\left(c_{1}\right)=v_{i}\left(c_{2}\right)=v_{i}\left(c_{1}, c_{2}\right)=y-\varepsilon>0$ and $v_{\hat{i}}\left(c_{1}\right)=v_{\hat{i}}\left(c_{2}\right)=v_{\hat{i}}\left(c_{1}, c_{2}\right)>0$.

In this case allocating both items to one player is not PO and allocating a singleton to player $i$ is not IR. Therefore, there cannot exist any mechanism that satisfies the properties with $y>0$. Thus we conclude $p\left(B_{i}\right)=0$.

3. $p\left(B_{i}=\left\{c_{1}, c_{2}\right\}\right)=0$

We proved in the above case that player i's price must be a constant. Suppose to the contrary that $p\left(B_{i}=\left\{c_{1}, c_{2}\right\}\right)=x>0$.

Consider the following valuations:

$v_{i}\left(c_{1}, c_{2}\right)=x-2 \varepsilon>0, v_{i}\left(c_{1}\right)=x-4 \varepsilon, v_{i}\left(c_{2}\right)=0, v_{\hat{i}}\left(c_{1}\right)=v_{\hat{i}}\left(c_{2}\right)=0, v_{\hat{i}}\left(c_{1}, c_{2}\right)=x-4 \varepsilon$.

From the definition of dictatorship and player i's DSIC the allocation must be $B_{i}=\left\{c_{1}\right\}$ as it maximizes i's utility (recall that we proved above $p\left(B_{i}\right)=0$ for this case). However, this allocation is not $P O$ as the allocation $B_{i}^{\prime}=\left\{c_{1}, c_{2}\right\}, p\left(B_{i}^{\prime}\right)=\varepsilon$ is strictly better for player $i$ and the auctioneer while player $\hat{i}$ is indifferent.

\section{Lemma C.1's Proof}

LEMMA C.1 In any nontrivial pricing mechanism that satisfies $I R, P O$, nonarbitrary hoarding and DSIC, stability must hold.

PROOF OF LEMMA 1 We consider two options:

1. $B_{i}=\left\{c_{1}, c_{2}\right\}$ and $B_{\hat{i}}=\emptyset$

Suppose to the contrary that player $i$ 's stability does not hold, i.e., $p\left(B_{i}\right)<\min \left\{b_{\hat{i}}, v_{\hat{i}}\left(c_{1}, c_{2}\right)\right\}$. Let $p\left(B_{i}\right)=\min \left\{b_{\hat{i}}, v_{\hat{i}}\left(c_{1}, c_{2}\right)\right\}-2 \cdot \varepsilon$.

Consider the same valuations for player $\hat{i}\left(V_{\hat{i}}^{\prime}=V_{\hat{i}}\right)$ and the following valuations for player $i$ :

- $v_{i}^{\prime}\left(c_{1}, c_{2}\right)=\min \left\{b_{\hat{i}}, v_{\hat{i}}\left(c_{1}, c_{2}\right)\right\}-\varepsilon$ and

- $v_{i}^{\prime}\left(c_{1}\right)=v_{i}^{\prime}\left(c_{2}\right)=0$

Then from the nonarbitrary hoarding property player $i$ will not be allocated the two-item bundle. Player $i$ 's utility in any other allocation is zero. Thus player $i$ is better off deviating and stating $V_{i}$ instead of $V_{i}^{\prime}$ and be allocated the two-item bundle for a positive utility. 
2. $B_{i}=\left\{c_{1}\right\}$ and $B_{\hat{i}}=\left\{c_{2}\right\}$

Suppose to the contrary that stability of player $i$ does not hold i.e., $p\left(B_{i}\right)<\min \left\{v_{\hat{i}}\left(c_{1}, c_{2}\right)-\right.$ $\left.v_{\hat{i}}\left(c_{2}\right), b_{\hat{i}}-p\left(B_{\hat{i}}\right)\right\}$. Let:

$p\left(B_{i}\right)=\min \left\{v_{\hat{i}}\left(c_{1}, c_{2}\right)-v_{\hat{i}}\left(c_{2}\right), b_{\hat{i}}-p\left(B_{\hat{i}}\right)\right\}-2 \cdot \varepsilon$.

Case I : $b_{\hat{i}}-p\left(B_{\hat{i}}\right) \geq v_{\hat{i}}\left(c_{1}, c_{2}\right)-v_{\hat{i}}\left(c_{2}\right)$

that is: $p\left(B_{i}\right)=v_{\hat{i}}\left(c_{1}, c_{2}\right)-v_{\hat{i}}\left(c_{2}\right)-2 \cdot \varepsilon$.

Consider the same valuations for player $\hat{i}\left(V_{\hat{i}}^{\prime}=V_{\hat{i}}\right)$ and the following valuations for player $i$ :

- $v_{i}^{\prime}\left(c_{2}\right)=0$

- $v_{i}^{\prime}\left(c_{1}, c_{2}\right)=v_{\hat{i}}\left(c_{1}, c_{2}\right)-v_{\hat{i}}\left(c_{2}\right)-\varepsilon$.

Note that, from $b_{\hat{i}}-p\left(B_{\hat{i}}\right) \geq v_{\hat{i}}\left(c_{1}, c_{2}\right)-v_{\hat{i}}\left(c_{2}\right)$ it follows that $v_{i}^{\prime}\left(c_{1}, c_{2}\right)<b_{\hat{i}}$.

- $v_{i}^{\prime}\left(c_{1}\right)=v_{i}^{\prime}\left(c_{1}, c_{2}\right)-\varepsilon / 2$

Allocating both items to player $i$ contradicts nonarbitrary hoarding as $v_{i}^{\prime}\left(c_{1}, c_{2}\right)<$ $\min \left\{b_{\hat{i}}, v_{\hat{i}}^{\prime}\left(c_{1}, c_{2}\right)\right\}$. Both allocations $\left(B_{i}^{\prime}=\left\{c_{2}\right\}, B_{\hat{i}}^{\prime}=\left\{c_{1}\right\}\right)$ and $\left(B_{\hat{i}}^{\prime}=\left\{c_{1}, c_{2}\right\}, B_{i}^{\prime}=\emptyset\right)$ are not DSIC as in both allocations player $i$ 's utility is 0 . Player $i$ can deviate and state $V_{i}$ instead of $V_{i}^{\prime}$. With this deviation he will be allocated $B_{i}^{\prime}=\left\{c_{1}\right\}$ for a positive utility, $u_{i}^{\prime}=v_{i}^{\prime}\left(c_{1}\right)-p\left(B_{i}\right)=\frac{\varepsilon}{2}>0$.

To complete the argument we now show that allocating $B_{\hat{i}}=\left\{c_{2}\right\}$ is not DSIC for player $\hat{i}$. Consider the following valuations: $V_{i}^{\prime \prime}=V_{i}^{\prime}, v_{\hat{i}}^{\prime \prime}\left(c_{2}\right)=0, v_{\hat{i}}^{\prime \prime}\left(c_{1}\right)=v_{\hat{i}}^{\prime}\left(c_{1}\right)=v_{\hat{i}}\left(c_{1}\right)$ and $v_{\hat{i}}^{\prime \prime}\left(c_{1}, c_{2}\right)=v_{\hat{i}}^{\prime}\left(c_{1}, c_{2}\right)+\varepsilon=v_{\hat{i}}\left(c_{1}, c_{2}\right)+\varepsilon$.

Allocating both items to player $i$ contradicts nonarbitrary hoarding as $v_{i}^{\prime \prime}\left(c_{1}, c_{2}\right)=$ $v_{i}^{\prime}\left(c_{1}, c_{2}\right)<v_{\hat{i}}\left(c_{1}, c_{2}\right)<v_{\hat{i}}^{\prime \prime}\left(c_{1}, c_{2}\right)$, and $v_{i}^{\prime \prime}\left(c_{1}, c_{2}\right)=v_{i}^{\prime}\left(c_{1}, c_{2}\right)<b_{\hat{i}}$.

Allocating singletons is not Pareto optimal as both players' valuation for $\left\{c_{2}\right\}$ is zero, and they both strictly prefer the two-item bundle over any singleton for the same price. Therefore by stating $V_{\hat{i}}^{\prime \prime}$ player $\hat{i}$ will be allocated the two-item bundle.

We first argue (below) that in any nontrivial pricing mechanism that satisfies the four properties, if player $\hat{i}$ is allocated the two-item bundle then $p\left(B_{\hat{i}}\right) \leq v_{i}\left(c_{1}, c_{2}\right)$. We then conclude that by stating $V_{\hat{i}}^{\prime \prime}$ player $\hat{i}$ will be allocated the two-item bundle for a price $p\left(B_{\hat{i}}^{\prime \prime}\right) \leq v_{i}^{\prime \prime}\left(c_{1}, c_{2}\right)$ and therefore the utility $u_{\hat{i}}^{\prime \prime}=v_{\hat{i}}\left(c_{1}, c_{2}\right)-p\left(B_{\hat{i}}^{\prime \prime}\right) \geq v_{\hat{i}}\left(c_{1}, c_{2}\right)-v_{i}^{\prime \prime}\left(c_{1}, c_{2}\right)=$ $v_{\hat{i}}\left(c_{2}\right)+\varepsilon$. That is, player $\hat{i}$ 's utility is higher than the utility from the allocation $B_{\hat{i}}=\left\{c_{2}\right\}$ where $c_{2}$ is allocated for free.

Proving the above argument: in any nontrivial pricing mechanism that satisfies the four properties, if player $\hat{i}$ is allocated the two-item bundle then $p\left(B_{\hat{i}}\right) \leq v_{i}\left(c_{1}, c_{2}\right)$.

Suppose to the contrary that $B_{\hat{i}}=\left\{c_{1}, c_{2}\right\}$ and $p\left(B_{\hat{i}}\right)=v_{i}\left(c_{1}, c_{2}\right)+2 \cdot \varepsilon$.

From nonarbitrary hoarding it must be the case that $\min \left\{b_{i}, v_{i}\left(c_{1}, c_{2}\right)\right\} \leq \min \left\{b_{\hat{i}}, v_{\hat{i}}\left(c_{1}, c_{2}\right)\right\}$.

Consider the valuations: $\bar{V}_{i}=V_{i} ; \bar{v}_{\hat{i}}\left(c_{1}, c_{2}\right)=v_{i}\left(c_{1}, c_{2}\right)+\varepsilon ; \bar{v}_{\hat{i}}\left(c_{1}\right)=\bar{v}_{\hat{i}}\left(c_{2}\right)=0$. 
Consider the allocation $\bar{B}_{i}=\left\{c_{1}, c_{2}\right\}$ :

$\min \left\{b_{i}, \bar{v}_{i}\left(c_{1}, c_{2}\right)\right\} \leq \min \left\{b_{\hat{i}}, \bar{v}_{\hat{i}}\left(c_{1}, c_{2}\right)\right\}$. Therefore this allocation does not contradict nonarbitrary hoarding only if:

i. $\bar{v}_{i}\left(c_{1}, c_{2}\right)=b_{\hat{i}} \leq b_{i}$

or:

ii. $b_{i}=b_{\hat{i}} \leq \bar{v}_{i}\left(c_{1}, c_{2}\right)$

However, both cases contradict IR for player $\hat{i}$ when the valuations are $V_{i}, V_{\hat{i}}$ as $p\left(B_{\hat{i}}\right)=$ $v_{i}\left(c_{1}, c_{2}\right)+2 \varepsilon>b_{\hat{i}}$.

If the allocation is $\bar{B}_{\hat{i}}=\left\{c_{1}, c_{2}\right\}$ then from IR it follows that $p\left(\bar{B}_{\hat{i}}\right) \leq \bar{v}_{\hat{i}}\left(c_{1}, c_{2}\right)=v_{i}\left(c_{1}, c_{2}\right)+$

$\varepsilon$. Therefore, when the true valuations are $V_{i}, V_{\hat{i}}$ then player $\hat{i}$ is better off deviating and stating $\bar{V}_{\hat{i}}$ to be allocated the same bundle for a lower price.

Therefore the mechanism must allocate singletons, and from IR player $\hat{i}$ must pay zero. However, in this case, if player $\hat{i}$ 's true valuations for the singletons are $\varepsilon$, he must still pay zero (from DSIC as his price cannot depend on his valuations). Thus, either the mechanism is a trivial pricing mechanism or it is not DSIC.

Case II $: b_{\hat{i}}-p\left(B_{\hat{i}}\right)<v_{\hat{i}}\left(c_{1}, c_{2}\right)-v_{\hat{i}}\left(c_{2}\right)$ that is, $p\left(B_{i}\right)<b_{\hat{i}}-p\left(B_{\hat{i}}\right)$. Note that if $v_{\hat{i}}\left(c_{1}, c_{2}\right) \leq b_{\hat{i}}$ then this case cannot happen as from IR it follows that $v_{\hat{i}}\left(c_{2}\right) \geq p\left(B_{\hat{i}}\right)$. From IR and DSIC we conclude that $p\left(B_{\hat{i}}\right)$ cannot be a constant unless $p\left(B_{\hat{i}}\right)=0$. The later case is a trivial pricing mechanism and therefore we do not claim that stability holds. From DSIC we also conclude that $p\left(B_{\hat{i}}\right)$ cannot be a function of player $\hat{i}$ 's valuations. Therefore $p\left(B_{\hat{i}}\right)$ must be a function of player $i$ 's valuations. This means that player $i$ has an incentive to raise $p\left(B_{\hat{i}}\right)$ and by doing so decrease the upper bound of $p\left(B_{i}\right)$ that he pays. This situation is not DSIC unless $p\left(B_{i}\right)=0$ (in which case player $i$ has no incentive to lower the price). In this case the mechanism is a trivial pricing mechanism and therefore stability does not hold. We therefore conclude that in case II the mechanism must be a trivial pricing mechanism.

\section{Proposition 3's Proof}

ProOf of PROPOSITION 3 We show that the prices cannot be higher than the lower bound given by stability and therefore they must equal the lower bound. We consider the two different cases.

1. $B_{i}=\left\{c_{1}, c_{2}\right\}$ and $B_{\hat{i}}=\emptyset$

Using IR we conclude that $p\left(B_{\hat{i}}\right)=0$.

Suppose to the contrary that $p\left(B_{i}\right)>\min \left\{b_{\hat{i}}, v_{\hat{i}}\left(c_{1}, c_{2}\right)\right\}$.

Let $p\left(B_{i}\right)=\min \left\{b_{\hat{i}}, v_{\hat{i}}\left(c_{1}, c_{2}\right)\right\}+2 \cdot \varepsilon$. According to IR it must be the case that $p\left(B_{i}\right)=\min \left\{b_{\hat{i}}, v_{\hat{i}}\left(c_{1}, c_{2}\right)\right\}+2 \cdot \varepsilon \leq \min \left\{b_{i}, v_{i}\left(c_{1}, c_{2}\right)\right\}$.

We show that a mechanism with such a price cannot be DSIC for player $i$.

Consider the following deviation for player $i$ :

- $v_{i}^{\prime}\left(c_{1}, c_{2}\right)=\min \left\{b_{\hat{i}}, v_{\hat{i}}\left(c_{1}, c_{2}\right)\right\}+\varepsilon$ and

- $v_{i}^{\prime}\left(c_{1}\right)=v_{i}^{\prime}\left(c_{2}\right)=0$ 
The allocation $B_{i}^{\prime}=\emptyset$ contradicts nonarbitrary hoarding as $\min \left\{b_{\hat{i}}, v_{\hat{i}}\left(c_{1}, c_{2}\right)\right\}+\varepsilon<\min \left\{b_{\hat{i}}, v_{\hat{i}}\left(c_{1}, c_{2}\right)\right\}+2 \varepsilon \leq \min \left\{b_{i}, v_{i}\left(c_{1}, c_{2}\right)\right\}$.

Suppose that each player is allocated a singleton: $B_{\hat{i}}^{\prime}=\left\{c_{j}\right\}$ and $B_{i}^{\prime}=\left\{c_{\hat{j}}\right\}$. Using the IR of player $i$ it follows that $p\left(B_{i}^{\prime}\right)=0$. Therefore, from Lemma C.1 it follows that $p\left(B_{\hat{i}}^{\prime}\right) \geq \min \left\{v_{i}^{\prime}\left(c_{1}, c_{2}\right)-v_{i}^{\prime}\left(c_{\hat{j}}\right), b_{i}-p\left(B_{i}^{\prime}\right)\right\}=\min \left\{v_{i}^{\prime}\left(c_{1}, c_{2}\right), b_{i}\right\}$. There are two cases:

Case I $v_{i}^{\prime}\left(c_{1}, c_{2}\right) \leq b_{i}$

From Lemma C.1 it follows that:

$p\left(B_{\hat{i}}^{\prime}\right) \geq v_{i}^{\prime}\left(c_{1}, c_{2}\right)=\min \left\{b_{\hat{i}}, v_{\hat{i}}\left(c_{1}, c_{2}\right)\right\}+\varepsilon$.

From free disposal we then conclude that: $p\left(B_{\hat{i}}^{\prime}\right) \geq \min \left\{b_{\hat{i}}, v_{\hat{i}}\left(c_{j}\right)\right\}+\varepsilon>\min \left\{b_{\hat{i}}, v_{\hat{i}}\left(c_{j}\right)\right\}$ with contradiction to IR of player $\hat{i}$.

Case II $v_{i}^{\prime}\left(c_{1}, c_{2}\right)>b_{i}$

However, in this case, $v_{i}^{\prime}\left(c_{1}, c_{2}\right)=\min \left\{b_{\hat{i}}, v_{\hat{i}}\left(c_{1}, c_{2}\right)\right\}+\varepsilon>b_{i}$ with contradiction to player i's IR, as our contrary assumption is that $p\left(B_{i}=\left\{c_{1}, c_{2}\right\}\right)=\min \left\{b_{\hat{i}}, v_{\hat{i}}\left(c_{1}, c_{2}\right)\right\}+2 \cdot \varepsilon>b_{i}$.

Therefore player $i$ must still be allocated the two-item bundle for a lower price. Thus any mechanism with a price higher than $\min \left\{b_{\hat{i}}, v_{\hat{i}}\left(c_{1}, c_{2}\right)\right\}$ is not DSIC.

2. $B_{1}=\left\{c_{j}\right\}$ and $B_{2}=\left\{c_{\hat{j}}\right\}$

- Suppose to the contrary that $p\left(B_{2}\right)>v_{1}\left(c_{1}, c_{2}\right)-v_{1}\left(c_{j}\right)$.

Let $p\left(B_{2}\right)=v_{1}\left(c_{1}, c_{2}\right)-v_{1}\left(c_{j}\right)+2 \cdot \varepsilon$. We show that any mechanism applying this price cannot be DSIC for player 2. Consider the following deviation for player 2:

$$
\begin{aligned}
& -v_{2}^{\prime}\left(c_{\hat{j}}\right)=v_{1}\left(c_{1}, c_{2}\right)-v_{1}\left(c_{j}\right)+\varepsilon \\
& \text { - } v_{2}^{\prime}\left(c_{j}\right)=0 \\
& \text { - } v_{2}^{\prime}\left(c_{1}, c_{2}\right)=v_{1}\left(c_{1}, c_{2}\right)+\frac{\varepsilon}{2}
\end{aligned}
$$

If $b_{1}<b_{2}$ then allocating both items to player 1 contradicts the property of nonarbitrary hoarding as $v_{2}^{\prime}\left(c_{1}, c_{2}\right)>v_{1}\left(c_{1}, c_{2}\right)$.

If $b_{1}=b_{2}$ from the property of nonarbitrary hoarding, as $v_{2}^{\prime}\left(c_{1}, c_{2}\right)>v_{1}\left(c_{1}, c_{2}\right)$, allocating both items to player 1 might satisfy the properties only if $v_{2}^{\prime}\left(c_{1}, c_{2}\right)>v_{1}\left(c_{1}, c_{2}\right) \geq b_{1}=b_{2}$. However, if this is the case then allocating both items to player 1 is not PO. Consider the following allocation: $B_{1}^{\prime \prime}=\left\{c_{j}\right\}, p\left(B_{1}^{\prime \prime}\right)=b_{1}-v_{2}^{\prime}\left(c_{\hat{j}}\right), B_{2}^{\prime \prime}=\left\{c_{\hat{j}}\right\}$ and $p\left(B_{2}^{\prime \prime}\right)=v_{2}^{\prime}\left(c_{\hat{j}}\right)$. Then player 2's utility is zero as before and the utility of the auctioneer is unchanged. Player 1's utility is strictly increased as $u_{1}^{\prime \prime}=v_{1}\left(c_{j}\right)-b_{1}+v_{2}^{\prime}\left(c_{\hat{j}}\right)=v_{1}\left(c_{j}\right)-b_{1}+v_{1}\left(c_{1}, c_{2}\right)-v_{1}\left(c_{j}\right)+\varepsilon>$ $v_{1}\left(c_{1}, c_{2}\right)-b_{1}=u_{1}^{\prime}$.

The allocation $B_{1}^{\prime}=\left\{c_{\hat{j}}\right\}$ is not IR for player 1 as from Lemma C.1 and from the free disposal assumption it follows that $p\left(B_{1}^{\prime}\right) \geq v_{2}^{\prime}\left(c_{1}, c_{2}\right)-v_{2}^{\prime}\left(c_{j}\right)=v_{1}\left(c_{1}, c_{2}\right)+\frac{\varepsilon}{2}>v_{1}\left(c_{1}, c_{2}\right) \geq v_{1}\left(c_{\hat{j}}\right)$. We claim that allocating both items to player 2 is not Pareto. We proved in case 1 above that if player 2 is allocated both items then the price must equal $\min \left\{b_{1}, v_{1}\left(c_{1}, c_{2}\right)\right\}$. Therefore, if on one hand $v_{1}\left(c_{1}, c_{2}\right) \leq b_{1}$ then player 2's utility from such an allocation is $\frac{\varepsilon}{2}$ and player 1's utility is zero. The allocation $B_{1}^{\prime \prime}=\left\{c_{j}\right\}, p\left(B_{1}^{\prime \prime}\right)=v_{1}\left(c_{j}\right), B_{2}^{\prime \prime}=\left\{c_{\hat{j}}\right\}$ and 
$p\left(B_{2}^{\prime \prime}\right)=v_{1}\left(c_{1}, c_{2}\right)-v_{1}\left(c_{j}\right)$ is strictly better for player 2 as $u_{2}^{\prime \prime}=v_{2}^{\prime}\left(c_{\hat{j}}\right)-v_{1}\left(c_{1}, c_{2}\right)+v_{1}\left(c_{j}\right)=$ $\varepsilon>\frac{\varepsilon}{2}=u_{2}^{\prime}$, while player 1 and the auctioneer are indifferent.

If on the other hand $v_{1}\left(c_{1}, c_{2}\right)>b_{1}$ then player 2's utility from such an allocation is $v_{1}\left(c_{1}, c_{2}\right)+\frac{\varepsilon}{2}-b_{1}$ and player 1's utility is zero. The allocation: $B_{1}^{\prime \prime \prime}=\left\{c_{j}\right\}, p\left(B_{1}^{\prime \prime \prime}\right)=v_{1}^{\prime}\left(c_{j}\right)$, $B_{2}^{\prime \prime \prime}=\left\{c_{\hat{j}}\right\}$ and $p\left(B_{2}^{\prime \prime \prime}\right)=b_{1}-v_{1}\left(c_{j}\right)$ is strictly better for player 2 as $u_{2}^{\prime \prime \prime}=v_{2}^{\prime}\left(c_{\hat{j}}\right)-b_{1}+$ $v_{1}\left(c_{j}\right)=v_{1}\left(c_{1}, c_{2}\right)+\varepsilon-b_{1}>v_{1}\left(c_{1}, c_{2}\right)+\frac{\varepsilon}{2}-b_{1}=u_{2}^{\prime}$ while player 1 and the auctioneer are indifferent.

Therefore player 2 must still be allocated $B_{2}=\left\{c_{\hat{j}}\right\}$ for a price lower than $v_{1}\left(c_{1}, c_{2}\right)-$ $v_{1}\left(c_{j}\right)+2 \cdot \varepsilon$. Thus any mechanism with a price higher than $v_{1}\left(c_{1}, c_{2}\right)-v_{1}\left(c_{j}\right)$ is not DSIC for player 2 .

- Suppose to the contrary that $p\left(B_{1}\right)>v_{2}\left(c_{1}, c_{2}\right)-v_{2}\left(c_{\hat{j}}\right)$.

Let $p\left(B_{1}\right)=v_{2}\left(c_{1}, c_{2}\right)-v_{2}\left(c_{\hat{j}}\right)+2 \cdot \varepsilon$. We claim that it is not truthful for player 1 . Consider the following deviation for player 1 :

$$
\begin{aligned}
& -v_{1}^{\prime}\left(c_{j}\right)=v_{2}\left(c_{1}, c_{2}\right)-v_{2}\left(c_{\hat{j}}\right)+\varepsilon \\
& \text { - } v_{1}^{\prime}\left(c_{\hat{j}}\right)=0 \\
& \text { - } v_{1}^{\prime}\left(c_{1}, c_{2}\right)=v_{2}\left(c_{1}, c_{2}\right)+\frac{\varepsilon}{2}
\end{aligned}
$$

From the property of nonarbitrary hoarding, as $v_{1}^{\prime}\left(c_{1}, c_{2}\right)>v_{2}\left(c_{1}, c_{2}\right)$, allocating both items to player 2 might satisfy the properties only if $v_{1}^{\prime}\left(c_{1}, c_{2}\right)>v_{2}\left(c_{1}, c_{2}\right) \geq b_{1}$. However, if this is the case then allocating both items to player 2 is not Pareto optimal. Consider the following allocation: $B_{1}^{\prime \prime}=\left\{c_{j}\right\}, p\left(B_{1}^{\prime \prime}\right)=v_{1}^{\prime}\left(c_{j}\right), B_{2}^{\prime \prime}=\left\{c_{\hat{j}}\right\}$ and $p\left(B_{2}^{\prime \prime}\right)=b_{1}-v_{1}^{\prime}\left(c_{j}\right)$. In this allocation player 1's utility is zero as before, the auctioneer's utility is unchanged, and player 2's utility is strictly increased as $u_{2}^{\prime \prime}=v_{2}\left(c_{\hat{j}}\right)-b_{1}+v_{1}^{\prime}\left(c_{j}\right)=v_{2}\left(c_{\hat{j}}\right)-b_{1}+v_{2}\left(c_{1}, c_{2}\right)-$ $v_{2}\left(c_{\hat{j}}\right)+\varepsilon>v_{2}\left(c_{1}, c_{2}\right)-b_{1}=u_{2}^{\prime}$.

The allocation $B_{2}^{\prime}=\left\{c_{j}\right\}$ is not IR for player 2, as from Lemma C.1 and the free disposal assumption it follows that $p\left(B_{2}^{\prime}\right) \geq v_{1}^{\prime}\left(c_{1}, c_{2}\right)-v_{1}^{\prime}\left(c_{\hat{j}}\right)=v_{2}\left(c_{1}, c_{2}\right)+\frac{\varepsilon}{2}>v_{2}\left(c_{1}, c_{2}\right) \geq v_{2}\left(c_{j}\right)$. Allocating both items to player 1 is not Pareto optimal. We proved in case 2 above that if player 1 is allocated both items then the price must equal $\min \left\{b_{2}, v_{2}\left(c_{1}, c_{2}\right)\right\}$. If $v_{2}\left(c_{1}, c_{2}\right) \leq b_{2}$ then player 1's utility from such an allocation is $\frac{\varepsilon}{2}$ and player 2 's utility is zero. The allocation $B_{1}^{\prime \prime \prime}=\left\{c_{j}\right\}, p\left(B_{1}^{\prime \prime \prime}\right)=v_{2}\left(c_{1}, c_{2}\right)-v_{2}\left(c_{\hat{j}}\right), B_{2}^{\prime \prime \prime}=\left\{c_{\hat{j}}\right\}$ and $p\left(B_{2}^{\prime \prime \prime}\right)=v_{2}\left(c_{\hat{j}}\right)$ is strictly better for player 1 as $u_{1}^{\prime \prime \prime}=v_{1}^{\prime}\left(c_{j}\right)-v_{2}\left(c_{1}, c_{2}\right)+v_{2}\left(c_{\hat{j}}\right)=\varepsilon>\frac{\varepsilon}{2}=u_{1}^{\prime}$. Player 2 and the auctioneer are indifferent. If $v_{2}\left(c_{1}, c_{2}\right)>b_{2}$ then the property of nonarbitrary hoarding implies that allocating both items to player 1 means $b_{1}=b_{2}$. Consider the following allocation: $\bar{B}_{1}=\left\{c_{j}\right\}, p\left(\bar{B}_{1}\right)=b_{1}-v_{2}\left(c_{\hat{j}}\right)$, $\bar{B}_{2}=\left\{c_{\hat{j}}\right\}$ and $p\left(\bar{B}_{2}\right)=v_{2}\left(c_{\hat{j}}\right)$. Then player 2's utility is zero as before, the auctioneer's utility is unchanged, and player 1 's utility is strictly increased as $\bar{u}_{1}=v_{1}^{\prime}\left(c_{j}\right)-b_{1}+v_{2}\left(c_{\hat{j}}\right)=v_{2}\left(c_{1}, c_{2}\right)-b_{1}+\varepsilon=v_{1}^{\prime}\left(c_{1}, c_{2}\right)-\frac{\varepsilon}{2}-b_{1}+\varepsilon>v_{1}^{\prime}\left(c_{1}, c_{2}\right)-b_{1}=u_{1}^{\prime}$.

Therefore any mechanism must still allocate $B_{1}=\left\{c_{j}\right\}$ for a price lower than $v_{2}\left(c_{1}, c_{2}\right)-$ $v_{2}\left(c_{\hat{j}}\right)+2 \cdot \varepsilon$. Thus any mechanism with a price higher than $v_{2}\left(c_{1}, c_{2}\right)-v_{2}\left(c_{\hat{j}}\right)$ is not DSIC for player 1 . 


\section{E. Implications of Pareto Optimality}

In the next claim we analyze the implications of the PO property on the allocation of the two-item bundle to one player. In the following section we use the implications of PO to show that there is no mechanism $F\left(V_{1}, V_{2}, b_{1}, b_{2}\right)$ that satisfies the four properties.

CLAIM 1 In any mechanism that satisfies the properties of IR, DSIC, PO, and nonarbitrary hoarding, if for a certain set of private valuations $V_{1}, V_{2}$ the output allocation is $B_{i}=\left\{c_{1}, c_{2}\right\}$ and $B_{\hat{i}}=\emptyset$, then $P O$ implies that $v_{i}\left(c_{1}, c_{2}\right)-v_{i}\left(c_{j}\right) \geq \min \left\{b_{\hat{i}}, v_{\hat{i}}\left(c_{3-j}\right)\right\}$ for every $j \in\{1,2\}$.

Proof of Claim 1 Suppose to the contrary that a mechanism $F$ satisfies the four properties, $B_{\hat{i}}=\emptyset$, $B_{i}=\left\{c_{1}, c_{2}\right\}$, but $v_{i}\left(c_{1}, c_{2}\right)-v_{i}\left(c_{2}\right)<\min \left\{b_{\hat{i}}, v_{\hat{i}}\left(c_{1}\right)\right\}$. Then from Proposition 3 we know that $p\left(B_{i}\right)=$ $\min \left\{b_{\hat{i}}, v_{\hat{i}}\left(c_{1}, c_{2}\right)\right\}$. Therefore $u_{i}=v_{i}\left(c_{1}, c_{2}\right)-\min \left\{b_{\hat{i}}, v_{\hat{i}}\left(c_{1}, c_{2}\right)\right\}$ and $u_{\hat{i}}=0$. The allocation $B_{\hat{i}}^{\prime}=\left\{c_{1}\right\}$ and $B_{i}^{\prime}=\left\{c_{2}\right\}$ with the prices $p\left(B_{\hat{i}}^{\prime}\right)=\min \left\{b_{\hat{i}}, v_{\hat{i}}\left(c_{1}\right)\right\}$ and $p\left(B_{i}^{\prime}\right)=\min \left\{b_{\hat{i}}, v_{\hat{i}}\left(c_{1}, c_{2}\right)\right\}-\min \left\{b_{\hat{i}}, v_{\hat{i}}\left(c_{1}\right)\right\}$ is strictly better for player $i$, better or equal for player $\hat{i}$, and equal for the auctioneer as:

- $u_{\hat{i}}^{\prime}=v_{\hat{i}}\left(c_{1}\right)-\min \left\{b_{\hat{i}}, v_{1}\left(c_{\hat{i}}\right)\right\} \geq 0=u_{\hat{i}}$

- $u_{i}^{\prime}=v_{i}\left(c_{2}\right)-\min \left\{b_{\hat{i}}, v_{\hat{i}}\left(c_{1}, c_{2}\right)\right\}+\min \left\{b_{\hat{i}}, v_{\hat{i}}\left(c_{1}\right)\right\}$. From the assumption that $v_{i}\left(c_{1}, c_{2}\right)-v_{i}\left(c_{2}\right)<$ $\min \left\{b_{\hat{i}}, v_{\hat{i}}\left(c_{1}\right)\right\}$ we conclude that $v_{i}\left(c_{1}, c_{2}\right)<v_{i}\left(c_{2}\right)+\min \left\{b_{\hat{i}}, v_{\hat{i}}\left(c_{1}\right)\right\}$. Thus, $u_{i}^{\prime}=v_{i}\left(c_{2}\right)-$ $\min \left\{b_{\hat{i}}, v_{\hat{i}}\left(c_{1}, c_{2}\right)\right\}+\min \left\{b_{\hat{i}}, v_{\hat{i}}\left(c_{1}\right)\right\}>v_{i}\left(c_{1}, c_{2}\right)-\min \left\{b_{\hat{i}}, v_{\hat{i}}\left(c_{1}, c_{2}\right)\right\}=u_{i}$.

- $p\left(B_{\hat{i}}\right)+p\left(B_{i}\right)=p\left(B_{\hat{i}}^{\prime}\right)+p\left(B_{i}^{\prime}\right)$.

This contradicts the assumption that the allocation is Pareto optimal. The proof that $v_{i}\left(c_{1}, c_{2}\right)-v_{i}\left(c_{1}\right) \geq$ $\min \left\{b_{\hat{i}}, v_{\hat{i}}\left(c_{2}\right)\right\}$ is equivalent.

\section{F. Impossibility Statement's Lemmas}

LEMMA F.1 If the following conditions hold:

1. $v_{2}\left(c_{1}, c_{2}\right)-v_{2}\left(c_{\hat{j}}\right)<\min \left\{b_{1}, v_{1}\left(c_{j}\right)\right\}$ and

2. $v_{1}\left(c_{1}, c_{2}\right)-v_{1}\left(c_{j}\right)<\min \left\{b_{2}, v_{2}\left(c_{\hat{j}}\right)\right\}$ and

3. $v_{2}\left(c_{1}, c_{2}\right)-v_{2}\left(c_{j}\right)>\min \left\{b_{1}, v_{1}\left(c_{\hat{j}}\right)\right\}$ and

4. $b_{1}-v_{2}\left(c_{1}, c_{2}\right)+v_{2}\left(c_{\hat{j}}\right)<v_{1}\left(c_{1}, c_{2}\right)-v_{1}\left(c_{j}\right)^{7}$

then any mechanism $F$ that satisfies the properties of IR, DSIC, PO, and nonarbitrary hoarding, must allocate $B_{1}=\left\{c_{j}\right\}, B_{2}=\left\{c_{\hat{j}}\right\}$ and $F$ must be a trivial pricing mechanism.

7 The motivation for Condition 4 is drawn from the proof of Lemma C.1 option 2 (case II), i.e. singletons allocation and $v_{i}\left(c_{1}, c_{2}\right)-v_{i}\left(B_{i}\right)>b_{i}-p\left(B_{i}\right)$. 
PROOF OF LEMMA 2 We first show that if Conditions 1-3 hold then any mechanism $F$ that satisfies the four properties must allocate $B_{1}=\left\{c_{j}\right\}$ and $B_{2}=\left\{c_{\hat{j}}\right\}$. Assume to the contrary that $F$ is a nontrivial pricing mechanism. From Claim 1 we conclude that Condition 1 implies that there is no mechanism that satisfies the four properties and allocates both items to player 2, and Condition 2 implies that there is no mechanism that satisfies the four properties and allocates both items to player 1. From Proposition 3 and Condition 3 it follows that allocating $B_{1}=\left\{c_{\hat{j}}\right\}$ is not IR for player 1. Therefore the allocation must be $B_{2}=\left\{c_{\hat{j}}\right\}$ and $B_{1}=\left\{c_{j}\right\}$.

From Proposition 3 we know that in any nontrivial pricing mechanism $p\left(B_{1}=\left\{c_{j}\right\}\right)=v_{2}\left(c_{1}, c_{2}\right)-$ $v_{2}\left(c_{\hat{j}}\right)$ and $p\left(B_{2}\right)=v_{1}\left(c_{1}, c_{2}\right)-v_{1}\left(c_{j}\right)$.

We showed that if Condition 4 holds then the mechanism must be a trivial pricing mechanism. From Condition 4 it follows that $u_{2}=v_{2}\left(c_{\hat{j}}\right)-v_{1}\left(c_{1}, c_{2}\right)+v_{1}\left(c_{j}\right)<v_{2}\left(c_{1}, c_{2}\right)-b_{1}$. Therefore player 2 will be better off deviating to be allocated the two-item bundle even for the highest price of $b_{1}$. Note that in any nontrivial pricing mechanism if $B_{2}=\left\{c_{1}, c_{2}\right\}$ then Proposition 3 implies that $p\left(B_{2}\right) \leq b_{1}$. Thus either there is no mechanism that satisfies the properties or the mechanism $F$ must be a trivial pricing mechanism.

LEMMA F.2 If the following conditions hold:

1. $v_{2}\left(c_{1}, c_{2}\right)-v_{2}\left(c_{\hat{j}}\right)<\min \left\{b_{1}, v_{1}\left(c_{j}\right)\right\}$ and

2. $v_{1}\left(c_{1}, c_{2}\right)-v_{1}\left(c_{j}\right)<\min \left\{b_{2}, v_{2}\left(c_{\hat{j}}\right)\right\}$ and

3. $v_{2}\left(c_{1}, c_{2}\right)-v_{2}\left(c_{j}\right)>\min \left\{b_{1}, v_{1}\left(c_{\hat{j}}\right)\right\}$ and

4. $b_{1}-v_{2}\left(c_{1}, c_{2}\right)+v_{2}\left(c_{\hat{j}}\right)>v_{1}\left(c_{1}, c_{2}\right)-v_{1}\left(c_{j}\right)$

then any mechanism $F$ that satisfies the properties of IR, DSIC, PO, and nonarbitrary hoarding must allocate $B_{1}=\left\{c_{j}\right\}$ and $B_{2}=\left\{c_{\hat{j}}\right\}$ with VCG prices.

PRoOF OF Lemma 3 In Lemma F.1 we showed that if Conditions 1-3 hold then any mechanism that satisfies the four properties must allocate $B_{1}=\left\{c_{j}\right\}$ and $B_{2}=\left\{c_{\hat{j}}\right\}$. In Lemma C.1 we showed that if the inequality in Condition 4 holds then the mechanism is a nontrivial pricing mechanism. From Proposition 3 we conclude that the prices must be VCG prices.

Though the nature of our result is an impossibility, Condition 4 both in Lemma F.1 and Lemma F.2 can provide insights into which valuation domains will allow the existence of efficient solutions and which domains will enforce inefficient solutions. For instance if Conditions 1-3 hold then Condition 4 enforces an efficient solution if $v_{1}\left(c_{1}, c_{2}\right) \leq b_{1}$. Additionally, Condition 4 together with Condition 1 establish the publicly-known budget $b_{1}$ as the threshold for efficiency.

\section{G. Theorem 1's Proof}

Proof of THEOREM 1 In Lemma F.1 we proved that for any mechanism $F$ that satisfies the four properties there are private valuations such that the mechanism must allocate $B_{1}=\left\{c_{j}\right\}$ and $B_{2}=\left\{c_{\hat{j}}\right\}$. Furthermore F in such cases is a trivial pricing mechanism. 
In Lemma F.2 we proved that for any mechanism $F$ that satisfies the four properties there are private valuations such that the mechanism must allocate $B_{1}=\left\{c_{j}\right\}$ and $B_{2}=\left\{c_{\hat{j}}\right\}$ with VCG prices.

Clearly, any mechanism in which a player can be allocated an item for free or be allocated the same item and pay the VCG price, cannot be DSIC. Therefore there cannot be a mechanism, F, that satisfies the four properties for any given private valuations.

\section{Acknowledgments}

The authors are grateful to the anonymous reviewers for their helpful comments, which significantly contributed to improving the quality of the publication.

\section{Conflicts of Interest}

The authors declare no conflict of interest.

\section{References}

1. Vickrey, W. Counterspeculation, auctions and competitive sealed tenders. J. Financ. 1961, 16, 8-37.

2. Clarke, E.H. Multipart pricing of public goods. Public Choice 1971, 2, 17-33.

3. Groves, T. Incentives in teams. Econometrica 1973, 41, 617-631.

4. Maskin, E.S. Auctions, development and privatization: Efficient auctions with liquidity-constrained buyers. Eur. Econ. Rev. 2000, 44, 667-681.

5. Lavi, R.; May, M. A note on the incompatibility of strategy-proofness and pareto-optimality in quasi-linear settings with public budgets. Econ. Lett. 2012, 115, 100-103.

6. Dobzinski, S.; Lavi, R.; Nisan, N. Multi-unit auctions with budget limits. Games Econ. Behav. 2008, 74, 486-503.

7. Fiat, A.; Leonardi, S.; Saia, J.; Sankowski, P. Single Valued Combinatorial Auctions with Budgets. In Proceedings of the 12th ACM Conference on Electronic Commerce EC, San Jose, CA, USA, June 5-9, 2011; pp. 223-232.

8. Bhattacharya, S.; Conitzer, V.; Munagala, K.; Xiax, L. Incentive Compatible Budget Elicitation in Multi-Unit Auctions. In Proceedings of the 21st Annual ACM-SIAM SODA, Austin, TX, USA, January 17-19, 2010; pp. 554-572.

9. Roberts, K. The Characterization of Implementable Choice Rules. In Aggregation and Revelation of Preferences; Laffont, J.J., Ed.; North-Holland Publishing Company: North-Holland, The Netherlands, 1979; pp. 321-348.

10. Green, J.; Laffont, J. Characterization of satisfactory mechanisms for the revelation of preferences for public goods. Econometrica 1977, 45, 427-438.

11. Holmstrom, B. Groves' scheme on restricted domains. Econometrica 1979, 47, 1137-1144.

12. Lehmann, D.; O'Callaghan, L.I.; Shoham, Y. Truth revelation in approximately efficient combinatorial auctions. J. ACM 2002, 49, 577-602.

13. Nisan, N.; Ronen, A. Computationally feasible VCG-based mechanisms. J. Artif. Intell. Res. 2007, $29,19-47$. 
14. Bartal, Y.; Gonen, R.; Nisan, N. Incentive Compatible Multi-Unit Combinatorial Auctions. In Proceedings of the 9th Theoretical Aspects of Rationality and KnowledgeTARK, Bloomington, IN, USA, June 20-22, 2003; pp. 72-87.

15. Dobzinski, S.; Nisan, N. Multi-Unit Auctions: Beyond Roberts. In Proceedings of the 12th ACM Conference on Electronic Commerce EC, San Jose, CA, USA, June 5-9, 2011; pp. 233-242.

16. Lerner, A.; Gonen, R. Dictatorial mechanisms in constrained combinatorial auctions. B.E. J.Theor. Econ. 2013, 13, doi: 10.1515/bejte-2013-0006.

17. Aggarwal, G.;Muthukrishnan, S.; Pa'l, D.; Pa'l, M. General Auction Mechanism for Search Advertising. In Proceedings of the 18th WWW '09, New York, NY, USA, April 20-24, 2009; pp. 241-250.

18. Ashlagi, I.; Braverman, M.; Hassidim, A.; Lavi, R.; Tennenholtz, M. Position auctions with budgets: Existence and uniqueness. B.E. J.Theor. Econ. 2010, 10, doi: 10.2202/1935-1704.1648.

19. Che, Y.K.; Gale, I. Standard auctions with financially constrained bidders. Rev. Econ. Stud. 1998, $65,1-21$.

20. Benoit, J.P.; Krishna, V. Multiple object auctions with budget constrained bidders. Rev. Econ. Stud. 2001, 68, 155-179.

21. Laffont, J.J.; Robert, J. Optimal auction with financially constrained buyers. Econ. Lett. 1996, 52, 181-186.

22. Pai, M. M.; Vohra, R. Optimal auctions with financially constrained bidders. Working paper 2008. Available online: http://www.econstor.eu/handle/10419/31205 (accessed on 15 November 2013).

(c) 2013 by the authors; licensee MDPI, Basel, Switzerland. This article is an open access article distributed under the terms and conditions of the Creative Commons Attribution license (http://creativecommons.org/licenses/by/3.0/). 